\title{
Association between Muscular Strength and Depressive Symptoms among Chinese Nursing Freshmen-A Cross-Sectional Study
}

Zhongyu Ren ( $\nabla$ renzhongyu@swu.edu.cn )

Southwest University https://orcid.org/0000-0002-7280-7669

Liya Guo

Southwest University

Jianhua Cao

Southwest University

Yingke Li

Southwest University

Guang Yang

Northeast normal university

Peng Cheng

Chongqing Nursing Vocational college

Bin Liu

Southwest univertisy

Zongji Hao

Southwest University

Hui Yao

Southwest University

Dongzhe Shi

Xinhua College of Sun Yat-Sen University

Chang Chen

Xinhua College of Sun Yat-Sen University

Li Peng

Southwest University

Research article

Keywords: Depressive symptoms, Muscular strength, College freshman, Chinese, Handgrip strength

Posted Date: December 4th, 2019

DOI: https://doi.org/10.21203/rs.2.18094/v1 
License: (c) (i) This work is licensed under a Creative Commons Attribution 4.0 International License. Read Full License 


\section{Abstract}

Increased physical activity level is related to lower risk of depressive symptoms, and an inverse association between muscular strength and risk of depressive symptoms among the elderly population. However, the association among younger adults is unclear. The aim of this study was to examine the association between muscle strength, using handgrip strength as a representative of skeletal muscle strength, and the risk of depressive symptoms among Chinese female college freshman. A crosssectional study was conducted among 867 participants aged between 16-23 years. Handgrip strength was measured with a handheld digital Smedley dynamometer, and depressive symptoms were evaluated using the 20-item Zung self-rating depression scale (SDS) with 3 cut-off points to indicate elevated depressive symptoms. We found that $10.7 \%$ of participants $(17.2 \%$ or $29.2 \%)$ were classified as having moderate to severe depressive symptoms using 50 as the cut-off point. After adjusting for these potential confounders, the adjusted odds ratios for moderate-to-severe depressive symptoms across tertile of relative handgrip strength were 1.00 (reference) for tertile $1,0.61$ (95\% $\mathrm{Cl}: 0.35,1.04)$ for tertile 2, and 0.53 ( $95 \%$ Cl: $0.39,0.94)$ for tertile 3 ( $P$ for trend: 0.024$)$. The significant associations remained when other cutoff points (SDS 48 or 45) were used. Interactions between the handgrip strength and potential confounders for depressive symptoms in the final models were insignificant. This study showed that handgrip strength is inversely and independently related to the risk of depressive symptoms among Chinese female college freshman. These results can help with the development of an effective intervention strategy against depression. For future research, it is important to explore the causality of the effects of handgrip strength on the depressive symptoms by intervention study.

\section{Introduction}

For college students, attending university for the first time is an important stage of life. The unfamiliarity of university life requires students to accomplish academic tasks and negotiate a new social world. Meanwhile, to facilitate their transition and adjustment to college life, many college freshmen may have to face academic and life stresses in today's competitive world. In this context, many college students experience psychological disturbances such as depression. Indeed, the National College Health Assessment in the United States found that approximately $53 \%$ of freshmen reported having experienced depression since beginning college ( 1 ). In Japan, $20 \%-30 \%$ of college freshmen reported having major depression (2). Similarly, the prevalence of depression or depressive symptoms among first-year university students in mainland China and Hong Kong was $24.8 \%$ and 43.9 , respectively (3). Although the prevalence of depressive symptoms among college freshmen in these studies differ from that of the general population (37.9\% for depressive symptoms) (4), which may be due to the use of different measurement tools, different methodologies, and different appraisal standards, previous studies have also found a general increase in mental health problems in college students $(5)$. Furthermore, it is well known that depression contributes to the onset of cancer (6), diabetes mellitus ( 7 ), coronary heart disease (7), stroke (8), and metabolic syndrome (9). At its worst, it can lead to suicide (10). Thus, the identification 
of potentially modifiable risk factors associated with the risk of depression is imperative to the development of an effective preventive strategy.

Although increasing levels of physical activity (PA) could protect against developing depression (11), only a few studies have examined objective measurements of physical fitness in relation to depression. Physical fitness (which includes both cardiorespiratory fitness and muscular strength) (12) may be a more objective risk factor, because improved physical fitness represents adaptation to regular PA (13). So far, an inverse association between cardiorespiratory fitness and risk of depression have been wellestablished among young (14) and middle-aged adults (15;16). Similarly, previous studies also reported an association between muscular strength and depression risk among the elderly population (17-19). However, to our knowledge, there have been no reports yet on whether muscle strength has a significant association with depression at younger ages. Elucidation of muscular strength at younger ages may help facilitate earlier and more effective preventive interventions.

Because handgrip strength is a simple, accurate and quick measurement of muscle strength, it can be a subrogation of general muscle strength (20). Moreover, handgrip strength is predictive of validity for mortality in older populations (21). To investigate the association between relative handgrip strength and the risk of depressive symptoms among the younger population, we designed a cross-sectional study in Chinese female college freshman.

\section{Materials And Methods}

\subsection{Design and samples}

The Chongqing Nursing Vocational College Physical Fitness and Health study is a prospective ongoing cohort study to assess the association between physical fitness and the health status of college students in Chongqing, China. Chongqing is the largest municipality of approximately 30.75 million inhabitants, located in the Southwest China.

Ethics approval was obtained from the ethical committee of the College of Physical Education of Southwest University (SWU20190101). A cross-sectional study was conducted to explore the association between muscular strength and depressive symptoms among Chinese nursing freshmen. In the period between October - December 2018 (baseline period), we recruited 1094 college freshmen at Chongqing Nursing Vocational College, who each participated in the annual physical fitness and completed a selfadministered questionnaire. This provided information about demographics, anthropometrics, lifestyle factors, and depressive status for data analysis. Written informed consent was obtained from all the participants or their parents or legal guardians for participants under the age of 16 . We excluded participants who did not complete the handgrip strength assessment $(n=59)$, as well as participants with missing information on age, height and/or body weight measurements, and PA $(n=62)$. Because of gender differences in handgrip strength, we found that the number of male participants $(n=106)$ were too small to perform a multiple logistic regression analysis and were therefore excluded. After the 
exclusions, the final sample of this study included 867 female participants between the age range of 16 23 years (mean 18.7, SD 1.0).

\subsection{Assessment of handgrip strength}

Assessment of handgrip strength was obtained using a dynamometer (EH101; CAMRY, Guangdong, China). All participants were told to adjust the dynamometer width for optimal hand comfort, and to relax their arm in a standing and stationary position. Each participant made four attempts using each hand with a brief interval between trials. The highest weight in kilograms of all handgrip strength measurements was used as a representative value of muscle strength. Handgrip strength relative to body weight $(\mathrm{kg} / \mathrm{kg}$ ) was calculated and examined as categorical variables in tertiles (low [0.32-0.50], medium [0.51-0.58], and high [0.59-0.94]).

\subsection{Assessment of depressive symptoms}

The Zung self-rating depression scale (SDS) was used to examine depression severity (22). It comprises of 20 items, and each item score ranges from 1 to 4, with a sum score between 20 to 80 . Higher scores represent a more severe depressive state. In order to increase the sensitivity of the detection and distinguish the severity of depressive symptoms, scores higher than these cut-off points $(45,48$, and 50$)$ reflect moderate or severe depressive symptoms $(23 ; 24)$. The reliability and validity of the Chinese version of SDS have been described in previous studies (25). In the present study, the Cronbach's a coefficient for the scale was 0.788 , which indicates that the Chinese version of the SDS in this study has a strong internal consistency.

\subsection{Relevant covariates}

The anthropometric variables (height and body weight) were measured using a standard protocol. Body mass index (BMI) was calculated as weight $(\mathrm{kg}) /$ height $^{2}\left(\mathrm{~m}^{2}\right)$. Demographic variables included: age (continuous variable), only child (yes or no), race (Han nationality, Tujia nationality, Miao nationality or other), father's educational level (senior high school or below, college or postgraduate), mother's educational level (senior high school or below, college or postgraduate), and parent's marital status (married, widowed or divorced). Lifestyle factors included: smoking status (never, occasionally or regularly), drinking status (never, occasionally or regularly), sleep duration (6-8 hour/d or less), and sleep quality (good or not) were assessed via a self-administered questionnaire. Levels of PA were assessed by the International Physical Activity Questionnaire (IPAQ) (short version) (26). Total weekly PA was calculated by metabolic equivalents $\times$ hour/week (26).

\subsection{Data analysis}

All continuous and categorical variables were presented as geometric least square mean ( $95 \%$ confidence interval) or proportions, and non-normal continuous variables were log-transformed for multivariate 
statistical analyses. For subject's characteristics, continuous variables were compared using analysis of variance (ANOVA), and categorical variables were compared by using chi-square tests.

Depressive symptoms were used as dependent variables and the tertiles of relative handgrip strength was used as independent variables. Multiple logistic regression analysis was also used to examine the relationship between tertiles of relative handgrip strength and depressive symptoms. Model 1 was a crude univariate model, while model 2 was adjusted for the relevant covariates. Interactions between handgrip strength levels and confounders of depressive symptoms were tested by the addition of crossproduct terms to the regression model. P-values $<0.05$ were considered statistically significant for all twosided tests. All tests were performed using the IBM SPSS Statistics 24.0 software (IBM SPSS Inc., Chicago, IL, USA).

\section{Results}

\subsection{Participants' characteristics according to tertiles of relative handgrip strength}

The subject's characteristics according to the tertile of relative handgrip strength are presented in Table 1. Compared to participants in the lowest tertile of relative handgrip strength, a lower proportion of participants in the upper two tertiles were obese $\left(\geq 30 \mathrm{~kg} / \mathrm{m}^{2}\right)$ or overweight $\left(\geq 25 \mathrm{~kg} / \mathrm{m}^{2}\right.$ and $<30 \mathrm{~kg} / \mathrm{m}^{2}$ ) ( $P$ for all trends $<0.05$ ). Excluding this, no significant difference was observed across the tertile of relative handgrip strength.

\subsection{Crude and adjusted associations of relative handgrip strength with depressive symptoms}

We analyzed the crude and adjusted relationship between the tertile of relative handgrip strength and total score for depressive symptoms. The total score for depressive symptoms differed significantly among tertiles of relative handgrip strength after adjustment for potential confounders. The mean ( $95 \%$ Cls) for the low, middle, and high groups were 40.9 (40.1-41.7), 40.2 (39.4-41.0), and 39.4 (38.6-40.2), respectively ( $P$ for trend: 0.011 ) (Figure 1 ).

3.3 Adjusted odds ratios (OR) (95\% confidence interval) of associations of relative handgrip strength with depressive symptoms

We also found that $10.7 \%$ ( $17.2 \%$ or $29.2 \%)$ of freshmen were classified as having moderate to severe depressive symptoms using 50 (48 or 45) as the cut-off point. Figure 2-4 shows the crude and adjusted significant relationship between the tertile of relative handgrip strength, and the risk of moderate-to-severe depressive symptoms. Using the 50 cut-off point in the final model, the adjusted ORs for moderate-tosevere depressive symptoms across tertiles of relative handgrip strength was 1.00 (reference) for tertile 1 , 0.61 (95\% Cl: $0.35,1.04)$ for tertile 2, and 0.53 (95\% Cl: $0.39,0.94)$ for tertile 3 ( $P$ for trend: 0.024$)$. Similar relationships were observed when using 48 or 45 as the cut-off point (Figure 4). Tests conducted to identify interactions between the tertile of relative handgrip strength and these potential confounders for depressive symptoms in the final models were not found to be significant. 


\section{Discussion}

A cross-sectional study was conducted among Chinese female college freshman to assess the relationship between handgrip strength and depressive symptoms. Multivariate logistic analyses have shown that higher handgrip strength level was significantly and independently associated with a lower risk of depressive symptoms after adjustment for potential confounders.

Currently, the relationship between higher handgrip strength (indicator of overall muscle strength), and lower risk of depressive symptoms is already widely accepted (17-19). However, this relationship had not been previously confirmed among younger adults. Our study expands on previous findings demonstrating that increased handgrip strength could be independently related to lows risk of depressive symptoms among college freshmen.

Although depressive symptoms are chronic and debilitating mood disorders, the exact etiology for depressive symptoms is unknown. We considered several plausible mechanisms. First, PA levels could mediate the inverse association between handgrip strength and depressive symptoms by lowering hypothalamic-pituitary-adrenocortical axis (HPA) reactivity to psychosocial stress, thus relieving depressive symptoms (27). In addition, physical activity may also reduce urine cortisol and epinephrine secretions (an indicator of sympathetic nerve activity in for psychophysical stress and depression) (28). In addition to physiological state, physical activity is also beneficial to one's psychological status. Regularly participating in physical activity can usually distract individuals from a negative or depressive mood to help cope with depression (29). Furthermore, perceived self-efficacy of physical activity may also play an important role in depressive moods. A randomized controlled trial-based meta-analysis demonstrated that physical activity is an effective method for improving perceived self-efficacy $(30)$. The theory of selfefficacy highlights the importance of self-regulation wherein higher level of perceived self-efficacy makes an individual more likely to initiate and maintain the behavior (31). In summary, regular physical activity could have a positive impact on depressive symptoms via physiological and psychological mechanisms. Although the significant relationship between handgrip strength and depressive symptoms remained after adjustment for PA level analyzed by the short version of IPAQ, other confounding PA types could still be associated with handgrip strength and depressive symptoms (26;32).

Second, Agudelo and colleagues found that depressive symptoms are related to elevated kynurenine levels, a tryptophan metabolite generated under stress (33). Activation of the skeletal muscle increases peroxisome proliferator-activated receptor-gamma co-activator-1 alpha 1 overexpression, promoting kynurenine aminotransferase expression, which likely prevents kynurenine from crossing the blood brain barrier to disrupt neural plasticity (34). Thus, improvement or maintenance of muscle strength may have a preventive effect against the risk of depressive symptoms.

Third, inflammatory biomarkers may also mediate the relationship between handgrip strength and risk of depressive symptoms. An adolescent-based study demonstrated that inflammatory proteins (C-reactive protein and IL-6) are inversely related to muscle strength (35). Inflammatory cytokines inhibit the 
production of insulin-like growth factor- 1 by the liver, which is an important anabolic stimulus for muscle (36). However, a positive relationship between inflammatory cytokine levels and depressive symptoms has also been shown (37). Studies have shown that a) activation of the inflammatory cytokine in depression can induce HPA-hyperactivity; b) cell-mediated immune activation may also contributes to disturbances of 5-hydroxytryptamine. Unfortunately, we were unable to provide evidence for kynurenine levels and inflammatory proteins. Future studies need to confirm whether kynurenine levels and inflammatory proteins mediate this relationship between handgrip strength and risk of depressive symptoms.

Furthermore, in this population-based study, we adjusted for a considerable number of confounding factors, which is especially important considering the complex etiology of depressive symptoms. Firstly, it is well known that the incidence of depressive symptoms and decline in skeletal muscle strength is increasing among Chinese college students (38;39). However, the association between handgrip strength and depressive symptoms remained, even after adjusting for age. It was reported that there was no significant association of depressive symptom with race (40), father's educational level (41), mother's educational level (41), parent's marital status (41), and being the only child (42). However, the adjustment of these socio-demographic variables did not have an effect on the significant association between handgrip strength and depressive symptoms. Second, greater muscle strength represents adaptation to regular physical activity, and physical activity may predict onset of depressive symptoms (11). Therefore, physical activity may play an intermediate role in the association between handgrip strength and depressive symptoms. However, additional adjustments for physical activity in our analysis did not alter our findings. Finally, adjusting the effect of weight (43), smoking (44), drinking (45) sleep duration (46), and sleep quality (47) on muscle strength did not change the significant association between handgrip strength and depressive symptoms. This suggests that handgrip strength is independently associated with depressive symptoms.

\subsection{Implications}

Previous studies investigating the association between muscle strength and the risk of depressive symptoms are widely based on epidemiologic studies, and the mechanism underlying this association is uncertain. The primary purpose of these studies were to provide specific exercise recommendations to prevent the development of depressive symptoms for improving public health. The current study further strengthens the evidence that greater muscle strength could be associated with a lower risk of depressive symptoms among the younger population, e.g., college students. Since each physical fitness component represents adaptation to regular physical activity, further prospective or intervention studies should identify whether each physical fitness component has a positive influence on depressive symptoms. These studies will provide insight regarding exercise recommendations that can be used by physicians and patients with depression, as well as provide information about the kind of sport activities that could help lower the risk of recurrent depression. The present study suggests that greater muscle strength is also associated with lower risk of depressive symptoms among younger population. 


\subsection{Limitations}

There are several limitations to our study. Firstly, since our study design was a population-based crosssectional study, the causation between handgrip strength and depressive symptoms could be not established. Secondly, although three cut-off points (SDS score: 45, 48 and 50) were used to define having moderate-to-severe depressive symptoms, we were unable to conduct a clinical diagnosis to determine the exact morbidity of depression. Thirdly, in the current study, the participants were all female freshmen. A meta-analysis including 39 studies and 32,694 Chinese college students showed no gender difference in prevalence of depressive symptoms (38). In addition, muscular strength is strongly associated with cardiorespiratory fitness (48), and there was an inverse association between cardiorespiratory fitness level and the risk of depressive symptoms (14). Inverse association similar to that in the current study seems validated. Nevertheless, further studies with a larger sample size of male college freshmen is necessary to confirm an association between muscle strength and the risk of depressive symptoms. Fourth, a longitudinal studies-based review showed that baseline depression may be a significant risk factor for the development of sedentary lifestyle or decreased level of physical activity (49). Thus, both bidirectional associations between muscle strength and depressive symptoms may exist. Further studies should take the causality and mechanism between the two into consideration. Finally, the results may not be representative of the Chinese general college freshman; therefore, further investigations with a larger sample size are needed to confirm our findings.

\section{Conclusions}

Our cross-sectional study assessed the relationship between handgrip strength and depressive symptoms in Chinese female college freshman. Increased physical activity level is an important factors to prevent the development of depressive symptoms, and measuring handgrip strength can be an indicator of muscle strength adaptation to regular physical activity. We demonstrated that an increased handgrip strength was significantly and independently related to lower risk of depressive symptoms. This can help with the development of an effective intervention strategy against depression. In future research, it is necessary to explore the causality between the effects of handgrip strength and depressive symptoms using intervention studies.

\section{Declarations}

\section{Conflict of Interest}

All the authors have no conflicts of interest exists to disclose.

\section{Author Contributions}

Conceived and designed the experiments: ZR LP CC. Performed the experiments: JC YL PC BL ZH HY DS. Analyzed the data: LG JC CC. Contributed reagents/materials/analysis tools: PC ZR. Wrote the paper: LG 
JC. Data collection: JC YL PC BL ZH HY DS.

\section{Funding}

This study was supported by the Fundamental Research Funds for the Central Universities (No. SWU1909734; SWU1709116; SWU1909105; 2412018JC015).

\section{Acknowledgments}

We thank Chongqing Nursing Vocational College freshmen who agreed to participate and gave informed consent for analysis of their data. We also would like to thank our staff from the Chongqing Nursing Vocational College and Southwest University for their dedicated work.

\section{References}

1 CACA analysis of the National College Health Assessment: reference group data base (American College Health Association, 2002)

2 Yamaguchi, N., ., Shimazaki, M., ., Hori, M., ., and Shiraishi, H. Early intervention and treatment of depressive and manic states among university students. Nihon Rinsho Japanese Journal of Clinical Medicine (1994) 52, 1383

3 Song, Y., Huang, Y., Liu, D., Kwan, J.S.H., Zhang, F., Sham, P.C., et al. Depression in college: depressive symptoms and personality factors in Beijing and Hong Kong college freshmen. Comprehensive Psychiatry (2008) 49, 496-502

4 Xuezheng Qin, S.W., Chee Ruey Hsieh The prevalence of depression and depressive symptoms among adults in China: Estimation based on a National Household Survey. China Economic Review (2018) 51, 271-282.doi:10.1016/j.chieco.2016.04.001

5 Liu, F., Zhou, N., Cao, H., Fang, X., Deng, L., Chen, W., et al. Chinese college freshmen's mental health problems and their subsequent help-seeking behaviors: A cohort design (2005-2011). Plos One (2017) 12.doi:10.1371/journal.pone.0185531

6 Jia, Y., Li, F., Liu, Y.F., Zhao, J.P., Leng, M.M., and Chen, L. Depression and cancer risk: a systematic review and meta-analysis. Public Health (2017) 149, 138-148

7 Poole, L., and Jackowska, M. The association between depressive and sleep symptoms for predicting incident disease onset after 6-year follow-up: findings from the English Longitudinal Study of Ageing. Psychological Medicine (2018) 49, 607-616 
8 Pan, A., Sun, Q., Okereke, O.I., Rexrode, K.M., and Hu, F.B. Depression and risk of stroke morbidity and mortality: a meta-analysis and systematic review. The Journal of the American Medical Association (2011) 306, 1241-1249

9 An, P., Nana, K., Olivia I, O., Qi, S., Mika, K., Richard R, R., et al. Bidirectional association between depression and metabolic syndrome: a systematic review and meta-analysis of epidemiological studies. Diabetes Care (2012) 36, 1171-1180

10 Rotenstein, L.S., Ramos, M.A., Torre, M., Segal, J.B., Peluso, M.J., Guille, C., et al. Prevalence of Depression, Depressive Symptoms, and Suicidal Ideation Among Medical Students: A Systematic Review and Meta-Analysis. Jama (2016) 316, 2214

11 Mammen, G., and Faulkner, G. Physical Activity and the Prevention of Depression : A Systematic Review of Prospective Studies. American Journal of Preventive Medicine (2013) 45, 649-657

12 Leong, D.P., Teo, K.K., Sumathy, R., Patricio, L.J., Alvaro, A., Andres, O., et al. Prognostic value of grip strength: findings from the Prospective Urban Rural Epidemiology (PURE) study. The Lancet (2015) 386, 266-273

13 Momma, H., Sawada, S.S., Kato, K., Gando, Y., Kawakami, R., Miyachi, M., et al. Physical Fitness Tests and Type 2 Diabetes Among Japanese: A Longitudinal Study From the Niigata Wellness Study. Journal of Epidemiology (2019) 29, 139-146.doi:10.2188/jea.JE20170280

14 Aberg, M.a.l., Waern, M., Nyberg, J., Pedersen, N.L., Bergh, Y., Aberg, N.D., et al. Cardiovascular fitness in males at age 18 and risk of serious depression in adulthood: Swedish prospective population-based study. British Journal of Psychiatry (2012) 201, 352-359.doi:10.1192/bjp.bp.111.103416

15 Sui, X., Laditka, J.N., Church, T.S., Hardin, J.W., Chase, N., Davis, K., et al. Prospective study of cardiorespiratory fitness and depressive symptoms in women and men. Journal of Psychiatric Research (2009) 43, 546-552

16 Dishman, R.K., Sui, X., Church, T.S., Hand, G.A., Trivedi, M.H., and Blair, S.N. Decline in cardiorespiratory fitness and odds of incident depression. American Journal of Preventive Medicine (2012) 43, 361-368

17 Norio, F., Yosuke, Y., Misa, T., Shin, Y., Yoshihiro, O., Miho, S., et al. Association between hand-grip strength and depressive symptoms: Locomotive Syndrome and Health Outcomes in Aizu Cohort Study (LOHAS). Age \& Ageing (2015) 44, 592-598

18 Wu, H., Yu, B., Meng, G., Liu, F., Guo, Q., Wang, J., et al. Both muscle mass and muscle strength are inversely associated with depressive symptoms in an elderly Chinese population. International Journal of Geriatric Psychiatry (2017) 32, 769-778

19 Smith, L., White, S., Stubbs, B., Hu, L., Veronese, N., Vancampfort, D., et al. Depressive symptoms, handgrip strength, and weight status in US older adults. Journal of Affective Disorders (2018) 238, 305- 
20 Wind, A.E., Takken, T., Helders, P.J.M., and Engelbert, R.H.H. Is grip strength a predictor for total muscle strength in healthy children, adolescents, and young adults? European Journal of Pediatrics (2010) 169, 281-287

21 Rijk, J.M., Roos, P.R., Deckx, L., Van, D.a.M., and Buntinx, F. Prognostic value of handgrip strength in people aged 60 years and older: A systematic review and meta-analysis. Geriatrics \& Gerontology International (2016) 16, 5-20

22 Zung, W.W. A self-rating depression scale. Archives of General Psychiatry (1965) 12, 63-70

23 Xu, L., Ren, J.M., Cheng, M., Tang, K.X., Dong, M., Hou, X.G., et al. Depressive symptoms and risk factors in Chinese persons with type 2 diabetes. Archives of Medical Research (2004) 35, 301307.doi:10.1016/j.arcmed.2004.04.006

$24 \mathrm{Su}, \mathrm{Q} ., \mathrm{Yu}, \mathrm{B} ., \mathrm{He}, \mathrm{H}$., Zhang, Q., Meng, G., Wu, H., et al. Nut consumption is associated with depressive symptoms among Chinese adults. Depression and Anxiety (2016) 33, 1065-1072.doi:10.1002/da.22516

25 Peng, H., Zhang, Y., Ying, G.I., Tang, W., Qiang, L.I., Yan, X., et al. Analysis of reliability and validity of Chinese version SDS Scale in women of rural area. Shanghai Medical \& Pharmaceutical Journal (2013) $34,20-23$

26 Craig, C.L., Marshall, A.L., Sjöström, M., Bauman, A.E., Booth, M.L., Ainsworth, B.E., et al. International physical activity questionnaire: 12-country reliability and validity. Medicine \& Science in Sports \& Exercise (2003) 35, 1381-1395

27 Stranahan, A.M., Lee, K., and Mattson, M.P. Central Mechanisms of HPA Axis Regulation by Voluntary Exercise. Neuromolecular Medicine (2008) 10, 118-127

28 Nabkasorn, C., Miyai, N., Sootmongkol, A., Junprasert, S., Yamamoto, H., Arita, M., et al. Effects of physical exercise on depression, neuroendocrine stress hormones and physiological fitness in adolescent females with depressive symptoms. European Journal of Public Health (2006) 16, 179

29 Bahrke, M.S., and Morgan, W.P. Anxiety reduction following exercise and meditation. Cognitive Therapy and Research (1978) 2, 323-333.doi:10.1007/bf01172650

30 Liu, M., Wu, L., and Ming, Q. How Does Physical Activity Intervention Improve Self-Esteem and SelfConcept in Children and Adolescents? Evidence from a Meta-Analysis. Plos One (2015) 10.doi:10.1371/journal.pone.0134804

31 Bandura, A. Social cognitive theory of self-regulation. Organizational Behavior and Human Decision Processes (1991) 50, 248-287.doi:https://doi.org/10.1016/0749-5978(91)90022-L 
32 Huang, C., Niu, K., Momma, H., Kobayashi, Y., Guan, L., Chujo, M., et al. Breakfast consumption frequency is associated with grip strength in a population of healthy Japanese adults. Nutrition, Metabolism and Cardiovascular Diseases (2014) 24, 648655.doi:https://doi.org/10.1016/j.numecd.2013.12.013

33 Moon, H.Y., and Van, P.H. Muscle over mind. Cell Metabolism (2014) 20, 560-562

34 Agudelo, L., Femenía, T., Orhan, F., Porsmyr-Palmertz, M., Goiny, M., Martinez-Redondo, V., et al. Skeletal Muscle PGC-1a1 Modulates Kynurenine Metabolism and Mediates Resilience to Stress-Induced Depression. Cell (2014) 159, 33-45

35 Vaara, J.P., Vasankari, T., ., Fogelholm, M., ., Häkkinen, K., ., Santtila, M., ., and Kyröläinen, H., . Maximal strength, muscular endurance and inflammatory biomarkers in young adult men. International Journal of Sports Medicine (2014) 35, 1229-1234

36 Ferrucci, L., Penninx, B.W.J.H., Volpato, S., Harris, T.B., Bandeen-Roche, K., Balfour, J., et al. Change in Muscle Strength Explains Accelerated Decline of Physical Function in Older Women With High Interleukin6 Serum Levels. Journal of the American Geriatrics Society (2002) 50, 1947-1954.doi:doi:10.1046/j.15325415.2002.50605.x

37 Yang, L., Roger Chun-Man, H., and Anselm, M. Interleukin (IL)-6, tumour necrosis factor alpha (TNF-a) and soluble interleukin-2 receptors (sIL-2R) are elevated in patients with major depressive disorder: a meta-analysis and meta-regression. Journal of Affective Disorders (2012) 139, 230-239

38 Lei, X.-Y., Xiao, L.-M., Liu, Y.-N., and Li, Y.-M. Prevalence of Depression among Chinese University Students: A Meta-Analysis. Plos One (2016) 11.doi:10.1371/journal.pone.0153454

39 Tian, Y., Jiang, C., Wang, M., Cai, R., Zhang, Y., He, Z., et al. BMI, leisure-time physical activity, and physical fitness in adults in China: results from a series of national surveys, 2000-14. Lancet Diabetes \& Endocrinology (2016) 4, 487-497.doi:10.1016/s2213-8587(16)00081-4

40 Fuhua Zhang, L.Z., Wen Zhang. Survey of Depression,Anxiety and Correlative Factors among Hinterland Minority Undergraduate Student. China Journal of Health Psychology (2016) 24, 12541257.doi:10.13342/j.cnki.cjhp.2016.08.037

41 Chen, L., Wang, L., Qiu, X.H., Yang, X.X., Qiao, Z.X., Yang, Y.J., et al. Depression among Chinese University Students: Prevalence and Socio-Demographic Correlates. Plos One (2013) 8.doi:10.1371/journal.pone.0058379

42 Yan Ting Rao, H.X.Z., Xiao Ning Li Impact of the Family Factors on College Students' Depression and Alienation. Psychological Development and Education (2004), 70-76.doi:10.16187/j.cnki.issn10014918.2004.01.014 
43 Keevil, V.L., Luben, R., Dalzell, N., Hayat, S., Sayer, A.A., Wareham, N.J., et al. Cross-sectional associations between different measures of obesity and muscle strength in men and women in a British cohort study. Journal of Nutrition Health \& Aging (2015) 19, 3-11.doi:10.1007/s12603-014-0492-6

44 Kok, M.O., Hoekstra, T., and Twisk, J.W.R. The Longitudinal Relation between Smoking and Muscle Strength in Healthy Adults. European Addiction Research (2012) 18, 70-75.doi:10.1159/000333600

45 Kawamoto, R., Ninomiya, D., Senzaki, K., and Kumagi, T. Alcohol Consumption is Positively Associated with Handgrip Strength Among Japanese Community-dwelling Middle-aged and Elderly Persons. International Journal of Gerontology (2018) 12, 294-298.doi:10.1016/j.ijge.2018.03.005

46 Wang, T.Y., Wu, Y., Wang, T., Li, Y., and Zhang, D. A prospective study on the association of sleep duration with grip strength among middle-aged and older Chinese. Experimental Gerontology (2018) 103, 88-93.doi:10.1016/j.exger.2018.01.009

47 Locquet, M., Beaudart, C., Delandsheere, L., Reginster, J.Y., and Bruyere, O. Subjective Sleep Quality among Sarcopenic and Non-Sarcopenic Older Adults: Results from the SarcoPhAge Cohort COHORT. Journal of Frailty \& Aging (2018) 7, 176-181.doi:10.14283/jfa.2018.13

48 Lindgren, M., Aberg, M., Schaufelberger, M., Aberg, D., Schioler, L., Toren, K., et al. Cardiorespiratory fitness and muscle strength in late adolescence and long-term risk of early heart failure in Swedish men. European Journal of Preventive Cardiology (2017) 24, 876-884.doi:10.1177/2047487317689974

49 Roshanaei-Moghaddam, B., Katon, W.J., and Russo, J. The longitudinal effects of depression on physical activity. General Hospital Psychiatry (2009) 31, 306-

315.doi:10.1016/j.genhosppsych.2009.04.002

\section{Table 1}

Table 1 Participants' characteristics according to tertiles of relative handgrip strength ${ }^{1}$ 


\begin{tabular}{|c|c|c|c|c|}
\hline & $\begin{array}{l}\text { Tertile } 1 \text { (low) } \\
(\mathrm{n}=286)\end{array}$ & $\begin{array}{l}\text { Tertile } \\
2 \\
(\mathrm{n} \\
=289)\end{array}$ & $\begin{array}{l}\text { tile } 3 \text { (high) } \\
=292 \text { ) }\end{array}$ & $\begin{array}{l}\text { P for } \\
\text { trend }^{2}\end{array}$ \\
\hline \multicolumn{5}{|l|}{ Demographic characteristics } \\
\hline Age, years & $\begin{array}{l}18.6(18.5 \\
18.7)\end{array}$ & $18.6(18.5,18.7)$ & $\begin{array}{l}18.7(18.6, \\
18.8)\end{array}$ & 0.278 \\
\hline \multicolumn{5}{|l|}{ Weight status, \% } \\
\hline$\geq 30 \mathrm{~kg} / \mathrm{m} 2$ & 1.0 & 0.0 & 0.0 & 0.047 \\
\hline $\begin{array}{l}\quad \geq 25 \mathrm{~kg} / \mathrm{m} 2 \text { and }<30 \mathrm{~kg} / \mathrm{m} 2 \\
\text { Smoking status, \% }\end{array}$ & 11.5 & 3.5 & 0.7 & $<0.001$ \\
\hline Occasionally & 5.6 & 5.2 & 3.4 & 0.424 \\
\hline Regularly & 0.3 & 0.3 & 0.3 & 1.000 \\
\hline \multicolumn{5}{|l|}{ Drinking status, \% } \\
\hline Occasionally & 55.9 & 46.4 & 48.6 & 0.056 \\
\hline Regularly & 0.0 & 1.7 & 1.4 & 0.097 \\
\hline \multicolumn{5}{|l|}{ Tertile of physical activity, \% } \\
\hline $\begin{array}{c}\text { Middle (range 28.43-58.50 } \\
\text { MET·h·week-1) }\end{array}$ & 32.2 & 34.3 & 33.6 & 0.864 \\
\hline High (range $58.50 \mathrm{MET} \cdot \mathrm{h} \cdot$ week-1) & 36.0 & 31.1 & 33.2 & 0.462 \\
\hline Sleep duration (6-8 hour), \% & 88.1 & 91.0 & 90.4 & 0.480 \\
\hline Sleep quality (good), \% & 75.2 & 74.7 & 77.1 & 0.788 \\
\hline \multicolumn{5}{|l|}{ Race, \% } \\
\hline Han nationality & 86.0 & 87.2 & 89.4 & 0.460 \\
\hline Tujia nationality & 9.4 & 7.3 & 6.5 & 0.392 \\
\hline Miao nationality & 2.8 & 3.5 & 1.7 & 0.416 \\
\hline \multicolumn{5}{|l|}{ Father education, \% } \\
\hline senior high school or less & 93.4 & 92.0 & 92.8 & 0.830 \\
\hline college & 6.3 & 8.0 & 6.8 & 0.729 \\
\hline \multicolumn{5}{|l|}{ Mother education, \% } \\
\hline Senior high school or less & 95.5 & 95.2 & 96.6 & 0.671 \\
\hline College & 4.5 & 4.8 & 3.4 & 0.671 \\
\hline \multicolumn{5}{|l|}{ Parent's marital status, $\%$} \\
\hline Widowed & 3.8 & 4.8 & 2.4 & 0.290 \\
\hline Divorced & 14.7 & 13.5 & 10.3 & 0.259 \\
\hline Only one child, \% & 27.6 & 26.6 & 21.6 & 0.198 \\
\hline
\end{tabular}

$\mathrm{PA}=$ physical activity $; \mathrm{BMI}=$ body mass index $\mathrm{MET}=$ metabolic equivalent

${ }^{1}$ Continuous variable without a normal distribution were log-transformed; Continuous variables are expressed as means (95\%CI) and categorical variables are expressed as percentages.

${ }^{2}$ Linear trends were assessed using analysis of variance for continuous variables and Chi-square test for categorical variables.

\section{Figures}




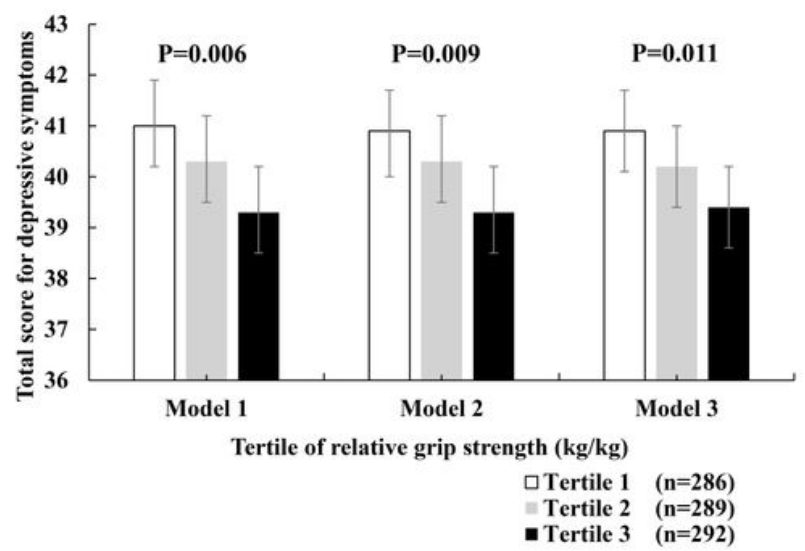

Figure 1. Crude and adjusted associations of relative handgrip strength with depressive symptoms among Chinese female college students. Model 1 are a crude univariate model; Model 2 adjusted for age (continuous variable) and BMI ( $\geq 30 \mathrm{~kg} / \mathrm{m}^{2}, \geq 25 \mathrm{~kg} / \mathrm{m}^{2}$ and $<30 \mathrm{~kg} / \mathrm{m}^{2}$, or $<25 \mathrm{~kg} / \mathrm{m}^{2}$ ); Model 3 additionally adjusted for race ( han nationality, tujia nationality, miao nationality and other nationality), only one child (yes or no), father education (senior high school or less, college and postgraduate), mother education (senior high school or less, college and postgraduate), smoking status (never smoker, former smoker and current smoker), drinking status (never drinker, former drinker and current drinker), physical activity level (low, middle, and high), sleep quality (good or not), sleep duration (6-8hour or not) and parent's marital status (married, widowed and divorced). Data are shown as means (total score for depressive symptoms) and $95 \%$ confidence intervals.

\section{Figure 1}

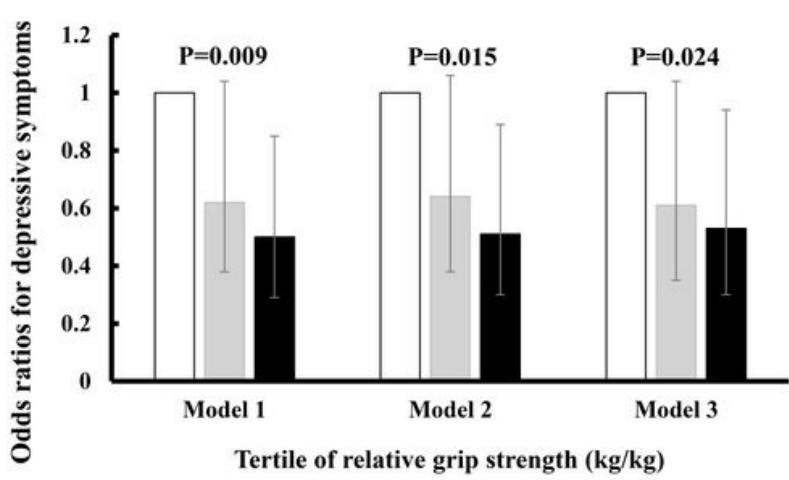

$\square$ Tertile $1 \quad(\mathrm{n}=\mathbf{2 8 6})$

Tertile $2 \quad(n=289)$

- Tertile $3 \quad(\mathrm{n}=\mathbf{2 9 2})$

Figure 2. Adjusted odds ratios ( $95 \%$ confidence interval) of associations of relative handgrip strength with depressive symptoms ( $S D S \geq 50$ ) among Chinese female college students. Model 1 are a crude univariate model; Model 2 adjusted for age (continuous variable) and BMI ( $\geq 30 \mathrm{~kg} / \mathrm{m} 2, \geq$ $25 \mathrm{~kg} / \mathrm{m} 2$ and $<30 \mathrm{~kg} / \mathrm{m} 2$, or $<25 \mathrm{~kg} / \mathrm{m} 2$ ); Model 3 additionally adjusted for race ( han nationality, tujia nationality, miao nationality and other nationality), only one child (yes or no), father education (senior high school or less, college and postgraduate), mother education (senior high school or less, college and postgraduate), smoking status (never smoker, former smoker and current smoker), drinking status (never drinker, former drinker and current drinker), physical activity level (low, middle, and high), sleep quality (good or not), sleep duration (6-8hour or not) and parent's marital status (married, widowed and divorced) 


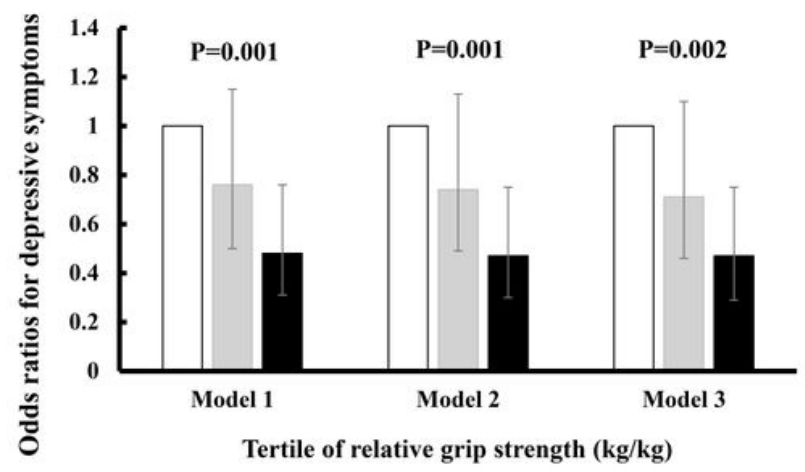

Tertile $1 \quad(\mathrm{n}=\mathbf{2 8 6})$

Tertile $2 \quad(\mathrm{n}=\mathbf{2 8 9})$

- Tertile $3 \quad(n=292)$

Figure 3. Adjusted odds ratios ( $95 \%$ confidence interval) of associations of relative handgrip strength with depressive symptoms (SDS $\geq 48$ ) among Chinese female college students. Model 1 are a crude univariate model; Model 2 adjusted for age (continuous variable) and BMI $(\geq 30 \mathrm{~kg} / \mathrm{m} 2, \geq$ $25 \mathrm{~kg} / \mathrm{m} 2$ and $<30 \mathrm{~kg} / \mathrm{m} 2$, or $<25 \mathrm{~kg} / \mathrm{m} 2)$; Model 3 additionally adjusted for race ( han nationality, tujia nationality, miao nationality and other nationality), only one child (yes or no), father education (senior high school or less, college and postgraduate), mother education (senior high school or less, college and postgraduate), smoking status (never smoker, former smoker and current smoker), drinking status (never drinker, former drinker and current drinker), physical activity level (low, middle, and high), sleep quality (good or not), sleep duration (6-8hour or not) and parent's marital status (married, widowed and divorced)

\section{Figure 3}

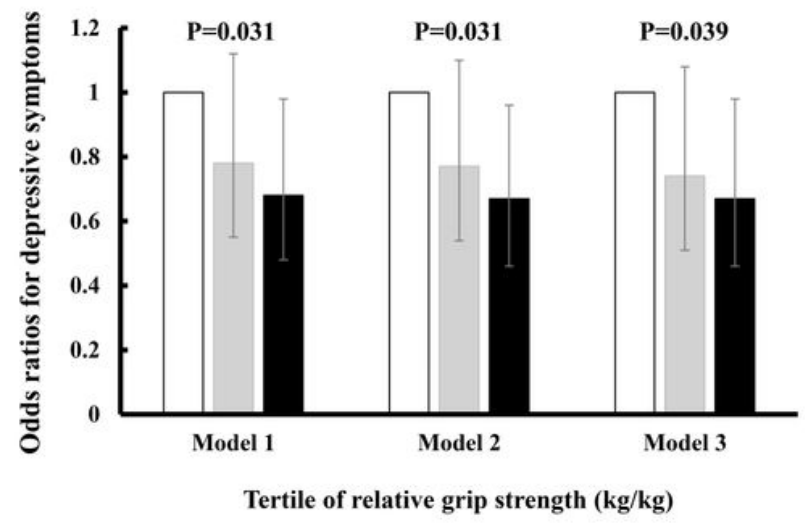

$\square$ Tertile $1 \quad(\mathrm{n}=286)$

Tertile $2 \quad(\mathrm{n}=\mathbf{2 8 9})$

- Tertile $3 \quad(\mathrm{n}=292)$

Figure 4. Adjusted odds ratios ( $95 \%$ confidence interval) of associations of relative handgrip strength with depressive symptoms ( $S D S \geq 45$ ) among Chinese female college students. Model 1 are a crude univariate model; Model 2 adjusted for age (continuous variable) and BMI $(\geq 30 \mathrm{~kg} / \mathrm{m} 2, \geq$ $25 \mathrm{~kg} / \mathrm{m} 2$ and $<30 \mathrm{~kg} / \mathrm{m} 2$, or $<25 \mathrm{~kg} / \mathrm{m} 2$ ); Model 3 additionally adjusted for race ( han nationality, tujia nationality, miao nationality and other nationality), only one child (yes or no), father education (senior high school or less, college and postgraduate), mother education (senior high school or less, college and postgraduate), smoking status (never smoker, former smoker and current smoker), drinking status (never drinker, former drinker and current drinker), physical activity level (low, middle, and high), sleep quality (good or not), sleep duration (6-8hour or not) and parent's marital status (married, widowed and divorced) 


\section{Supplementary Files}

This is a list of supplementary files associated with this preprint. Click to download.

- Stables.docx 